$\underline{\xi}=$ 离

\title{
You can't b. cereus: an unusual presentation of bacillus cereus
}

\author{
Laura Hamant ${ }^{1}$, Farmin Samareh-Jahani ${ }^{1}$ *, Paul Muna Aguon MD ${ }^{2}$, Brenen Swofford DO ${ }^{2}$, \\ Thomas Marston MD ${ }^{2}$, Alisha Nanda MD ${ }^{2}$, Julie Crawford MD ${ }^{3}$ \\ ${ }^{1}$ University of Arizona College of Medicine - Phoenix, 550 E. Van Buren Street, Phoenix, AZ 85004 \\ ${ }^{2}$ Banner University Medical Center - Phoenix, 1111 E McDowell Road, Phoenix, AZ 85006 \\ ${ }^{3}$ Carl T. Hayden VA Medical Center, 650 E Indian School Road, Phoenix, AZ 85012 \\ *Corresponding author E-mail: samarehj@email.arizona.edu
}

\begin{abstract}
We present a case of a 74-year-old male with a complicated medical history who was admitted to the medical floor for evaluation and management of failure to thrive and malnutrition. On hospital day 9 he became febrile and blood cultures were found to be positive for B. cereus and Enterobacter cloacae, which were persistent. He had an extensive negative workup for the cause of his bacteremia, however, we present a number of possibilities from his presentation and a literature review. Our case was consistent with other cases in the literature as our patient could be considered immunocompromised secondary to malnutrition and type II diabetes. B. cereus in blood cultures is often considered a contaminant but can be true bacteremia and should be worked up if multiple blood cultures are positive or if there is a clinical suspicion.
\end{abstract}

Keywords: Bacillus Cereus; B. Cereus; Bacteremia; Immunocompromised; Type II Diabetes.

\section{Introduction}

Bacillus cereus is a gram-positive aerobic or facultatively anaerobic, motile, spore-forming, rod-shaped bacteria that is widely distributed across environments and relatively protected in its endospore form. This organism is most commonly associated with gastroenteritis from reheated rice, causing self-limited vomiting and/or diarrhea. However, it is often considered a contaminant when isolated in blood cultures.

B. cereus has the capability to cause systemic and local infections for both immunocompromised and immunocompetent individuals as evidenced by a review of the literature. Previous case reports show catheters as being considered a primary cause for B. cereus bloodstream infections [2], [4]. In regard to urinary catheter related infections, the authors only found one case report of a B. cereus urinary tract infection ${ }^{5}$. B. cereus can also be a rare cause of endocarditis, typically associated with intravenous drug abuse, prosthetic valves, or pacemakers. However, there is a case that documents a patient who suffered from native valve B. cereus endocarditis with no apparent risk factors ${ }^{7}$. Other case reports of atypical B. cereus bacteremia have been documented secondary to pneumonia, with one demonstrating an elderly, immunocompetent woman contracting B. cereus while being managed for congestive heart failure [6].

\section{Case}

A 74-year-old male with history of type II diabetes mellitus, heart failure with reduced ejection fraction, atrial fibrillation on anticoagulation, neurofibromatosis, and abdominal aortic aneurysm with an endovascular aneurysm repair (EVAR) graft was admitted to the medical floor for management of failure to thrive and malnu- trition. Physical exam revealed a cachectic elderly man with poor dentition, irregularly irregular heart rhythm, and diffuse dermal neurofibromas. Labs showed WBC 11, K 2.4, Cr 1.49, and INR 7.0. Chest radiograph was negative for acute pathology.

On hospital day two, the patient developed diarrhea, but his stool was negative for Clostridium Difficile, ova and parasites, or other bacterial pathogens. He was continued on a higher rate of IV fluids and his diarrhea eventually resolved. Nine days into his hospitalization, he became febrile to $101.4^{\circ} \mathrm{F}$ with leukocytosis (WBC 14.5) and subsequent blood and urine cultures were collected. Blood cultures were positive for Enterobacter cloacae and B. cereus in 2 out of 2 sets. Urine cultures grew mixed gram positive and gram-negative urogenital microbiota. The patient was started on vancomycin and ceftriaxone resulting in the Enterobacter cloacae being cleared by the second blood cultures drawn on day three. However, B. cereus was persistent in blood cultures for six days despite normalizing leukocytes. Chest imaging was able to detect a left lower lobe infiltrate but without associated symptoms of cough or sputum production. No other obvious source was elucidated for the persistent B. cereus bacteremia after PET scan revealed a non-infected EVAR endograft, a negative CT abdomen/pelvis, and a transesophageal echocardiogram (TEE) without signs of vegetation or other intracardiac source of infection. Given his clinical improvement, he was discharged on levofloxacin for suspected hospital acquired pneumonia from the date of negative cultures, with plans for close follow up.

\section{Discussion}

This case proves interesting because of the unusual presentation of B. cereus bacteremia. Most case reports and series of B. cereus bacteremia point to immunocompromised status by means of hematologic disease or through immunosuppression therapy after 
transplant. Although not by conventional means, we could consider our patient immunocompromised secondary to his malnutrition complicated by type II diabetes. Even so, his leukocyte count remained within normal limits or elevated for the majority of his stay in the hospital. With a diffusely negative work up including CT abdomen/pelvis, PET scan, EGD, and TEE, in addition to his medical or social history lacking any prominent clues, some possibilities for the etiology of his bacteremia are hospital acquired pneumonia or multiple venous catheters during his hospital stay. One possibility is that his diarrhea was actually B. cereus gastroenteritis which then translocated along with Enterobacter cloacae. Another possibility is that despite the negative PET scan, his EVAR could have been seeded with B. cereus causing the persistent bacteremia.

Most practitioners regard B. cereus isolated from blood cultures and open wounds as contaminants. However, having repeated blood culture testing positive for B. cereus and with no reported increase in the incidence of B. cereus among other blood cultures from the microbiology lab, this scenario became less likely. B. cereus can cause true bacteremia and if multiple blood cultures are positive or if there is a clinical suspicion, it should be further investigated. For future consideration, B. cereus isolates should be forwarded to a reference center for genotypic fingerprinting and/or serotyping to determine whether there is a local source of contamination [3].

\section{References}

[1] Bottone EJ. Bacillus cereus, a volatile human pathogen. Clin $\mathrm{Mi}$ crobiol Rev. 2010; 23(2):382-398. https://doi.org/10.1128/CMR.00073-09.

[2] Kuroki R, Kawakami K, Qin L, et al. Nosocomial bacteremia caused by biofilm-forming bacillus cereus and bacillus thuringiensis. Intern Med. 2009; 48(10):791-796. https://doi.org/10.2169/internalmedicine.48.1885.

[3] Liu PY, Ke SC, Chen SL. Use of pulsed-field gel electrophoresis to investigate a pseudo-outbreak of bacillus cereus in a pediatric unit. J Clin Microbiol: 1997; 35(6):1533-1535. https://doi.org/10.1016/j.jhin.2006.05.014.

[4] Ozkocaman V, Ozcelik T, Ali R, et al. Bacillus spp. Among hospitalized patients with haematological malignancies: Clinical features epidemics and outcomes. J Hosp Infect. 2006; 64(2):169-176.

[5] Sato K, Ichiyama S, Ohmura M, et al. A case of urinary tract infection caused by bacillus cereus. J Infect. 1998; 36(2):247-248. https://doi.org/10.1016/S0163-4453(98)80032-7.

[6] Shimoyama, Y. et al. Bacillus cereus pneumonia in an immunocompetent patient: A case report. JA Clinical Reports. 2017; 3:25 https://doi.org/10.1186/s40981-017-0096-3.

[7] Thomas BS, Bankowski MJ, Lau WKK. Native Valve Bacillus cereus Endocarditis in a Non-Intravenous-Drug-Abusing Patient Journal of Clinical Microbiology. 2012; 50(2):519-521. https://doi.org/10.1128/JCM.00657-11. 